\title{
Lessons from the Chelyabinsk event
}

\author{
Vacheslav V. Emel'yanenko \\ Institute of Astronomy, Russian Academy of Sciences, \\ 48 Pyatnitskaya str., 119017, Moscow, Russia \\ email: vvemel@inasan.ru
}

\begin{abstract}
Recent investigations on small asteroids, initiated by the Chelyabinsk event, are reviewed. New estimates of the terrestrial impact rate, importance of Sun-grazing conditions in the evolution of near-Earth objects, and problems associated with dangerous objects approaching the Earth from the Sun direction are discussed.
\end{abstract}

Keywords. celestial mechanics, meteors, meteoroids, minor planets, asteroids

\section{Introduction}

After the Chelyabinsk event, it is evident that not only large asteroids but also $\sim 10$ $\mathrm{m}$ size bodies pose a substantial hazard to the Earth civilization. Although the number of near-Earth objects has been growing rapidly in this century due to dedicated surveys, there are large uncertainties in the population count, physical properties and dynamical features of small asteroids. Some new results in addressing these issues, initiated by the Chelyabinsk event, are reviewed here.

\section{Distribution of near-Earth objects}

After the Chelyabinsk event, Brown et al. (2013) confirmed earlier infrasonic influx estimates (Silber et al. 2009) that the bolide impactor flux at the Earth is an order of magnitude higher than estimates based on telescopic surveys. This prompted Harris \& D'Abramo (2015) to consider again the size distribution of near-Earth objects. The new estimated frequences of the impacts are substantially higher than earlier results. Nevertheless, there are still differences between the data obtained from the bolide statistics and the near-Earth asteroid surveys. While Harris \& D'Abramo (2015) predict that the Tunguska-sized body impact and the Chelyabinsk-sized body impact should occur about once in 500 years and 50 years, correspondingly, Brown et al. (2013) give 80-210 years and 20 years for these events.

There are problems not only on numbers of small asteroids, but also about distributions of their sizes and dynamical characteristics. In particular, the dip in the size distribution curve around $100 \mathrm{~m}$ is a long-standing problem. Harris \& D'Abramo (2015) suggest that this may correspond to a transition from weak, rubble-pile bodies to stronger, monolithic small asteroids. One more feature is associated with an unusual dependence of mean velocities with respect to the Earth on absolute magnitudes of small objects approaching the Earth (Emel'yanenko \& Naroenkov 2015).

\section{Origin of small asteroids near the Sun}

The classical interpretation for the origin of small near-Earth objects is a catastrophic disruption event through a major collision within the main belt. But very short cosmic 
ray exposure (CRE) ages for some meteorites are inconsistent with their origin from catastrophic disruption within the main belt (Connolly et al. 2015). The Chelyabinsk meteorite has added one more example to the set of short CRE ages. The estimates of its CRE age range from 1.2 Myr (Popova et al. 2013, Povinec et al. 2015) to $1.6 \mathrm{Myr}$ (Nishiizumi et al. 2013). This implies the origin of Chelyabinsk as a free-floating body in the near-Earth region.

A sudy of the dynamical evolution of the Chelyabinsk object has shown that there exists a large probability that the Chelyabinsk object was near the Sun in the past (Emel'yanenko et al. 2014). The most probable time of the encounter with the Sun lies in the interval from $0.8 \mathrm{Myr}$ to $2 \mathrm{Myr}$. This is consistent with the estimates of the Chelyabinsk CRE age. It is natural to assume that tidal and thermal effects could lead to disruption of a larger parent body near the Sun.

It is well known from dynamical studies that near-Earth objects evolve frequently to orbits with small perihelion distances (Farinella et al. 1994, Gladman et al. 2000, Foschini et al. 2000, Marchi et al. 2009). It is estimated that up to 70 percent of near-Earth objects collide with the Sun during their orbital evolution (Marchi et al. 2009). Thus, disruption of bodies due to the strong solar tide, thermal stresses and interaction with the solar atmosphere at Sun-grazing conditions may play a key role in the origin and modification of small near-Earth objects. Now theoretical and experimental works on the physical changes of asteroids near the Sun are clearly insufficient.

\section{Day-time impactors}

It is known that the Chelyabinsk object came from the sunward direction. The number of observed daytime bolides is almost equal to the number of observed nighttime bolides (http://neo.jpl.nasa.gov/jpl/bolide-events-1994-2013). Thus, discovery of dangerous objects approaching the Earth from the Sun direction is a very serious task in the asteroid hazard problem. A dedicated space system is the only way for us to be warned about threatening bodies that come to the Earth from the day sky. In this coonnection, a special project of space telescopes located near the $L_{1}$ point is developed in Russia (Shustov et al. 2015 ).

\section{Acknowledgements}

This work was supported by the RAS Presidium Program 9.

\section{References}

Brown, P. G., Assink, J. D., Astiz, L., et al. 2013, Nature, 503, 238

Connolly, H. C., Lauretta, D. S., Walsh, K. J., Tachibana, S., \& Bottke, W. F. 2015, Earth, Planets and Space, 67, id. 12

Emel'yanenko, V. V., Naroenkov, S. A., Jenniskens, P., \& Popova, O. P. 2014, MEPS, 49, 2169

Emel'yanenko, V. V. \& Naroenkov, S. A. 2015, Astrophysical Bulletin, 70, 342

Farinella, P., Froeschle, Ch., Froeschle, Cl., Gonczi, R., Hahn, G., Morbidelli, A., \& Valsecchi, G. B. 1994, Nature, 371, 314

Foschini, L., Farinella, P., Froeschle, Ch., Gonczi, R., Jopek, T. J., \& Michel, P. 2000, A\&A, 353,797

Gladman, B., Michel, P., \& Froeschle, Ch. 2000, Icarus, 146, 176

Harris, A. W. \& D'Abramo, G. 2015, Icarus, 257, 302

Marchi, S., Delbo, M., Morbidelli, A., Paolicchi, P., \& Lazzarin, M. 2009, MNRAS, 400, 147 
Nishiizumi, K., Caffee, M. W., Huber, L., Welten, K. C., \& Wieler, R. 2013, MESS Supplement, id. 5260

Popova, O. P., Jenniskens, P., Emel'yanenko, V., et al. 2013, Science, 342, 1073

Povinec, P. P., Laubenstein, M., Jull, A. J. T., et al. 2015, MESPS, 50, 273

Shustov, B. M., Shugarov, A. S., Naroenkov, S. A., \& Prokhorov, M. E. 2015, Astronomy Reports, 59 , No. 10 , in press

Silber, E. A., ReVelle, D. O., Brown, P. G., \& Edwards, W. N. 2009, Journal of Geophysical Research, 114, E08006 